\title{
Maclaurin's series expansions of real powers of inverse (hyperbolic) cosine and sine functions with applications
}

Feng Qi ( $\sim$ qifeng618@gmail.com)

https://orcid.org/0000-0001-6239-2968

\section{Research Article}

Keywords: Maclaurin's series expansion, real power, inverse cosine function, inverse hyperbolic cosine function, inverse sine function; inverse hyperbolic sine function, Stirling number of the first kind, Bell polynomial of the second kind, Faa di Bruno formula, central binomial coefficient, combinatorial identity, infinite series representation, $\mathrm{Pi}$

Posted Date: October 14th, 2021

DOI: https://doi.org/10.21203/rs.3.rs-959177/v2

License: (c) (i) This work is licensed under a Creative Commons Attribution 4.0 International License. Read Full License 


\title{
MACLAURIN'S SERIES EXPANSIONS OF REAL POWERS OF INVERSE (HYPERBOLIC) COSINE AND SINE FUNCTIONS WITH APPLICATIONS
}

\author{
FENG QI
}

Dedicated to people facing and battling COVID-19

\begin{abstract}
In the paper, by means of the Faà di Bruno formula, with the help of explicit formulas for special values of the Bell polynomials of the second kind with respect to a specific sequence, and by virtue of two combinatorial identities containing the Stirling numbers of the first kind, the author establishes Maclaurin's series expansions for real powers of the inverse cosine (sine) function and the inverse hyperbolic cosine (sine) function. By applying different series expansions for the square of the inverse cosine function, the author not only finds infinite series representations of the circular constant $\mathrm{Pi}$ and its square, but also derives two combinatorial identities involving central binomial coefficients.
\end{abstract}

\section{Contents}

1. Motivations

2. Explicit formulas for special values of Bell polynomials of second kind

3. Maclaurin's series expansions for real powers of inverse cosine function

4. Maclaurin's series expansions for real powers of inverse sine function

5. Infinite series representations related to $\mathrm{Pi}$ and its square

6. Two combinatorial identities

7. Remarks

8. Declarations

References

\section{Motivations}

The classical Euler gamma function $\Gamma(z)$ can be defined [26, Chapter 3] by

$$
\Gamma(z)=\lim _{m \rightarrow \infty} \frac{m ! m^{z}}{\prod_{k=0}^{m}(z+k)}, \quad z \in \mathbb{C} \backslash\{0,-1,-2, \ldots\} .
$$

The modified Bessel function of the first kind $I_{\nu}(z)$ can be represented [1, p. 375, 9.6.10] by

$$
I_{\nu}(z)=\sum_{n=0}^{\infty} \frac{1}{n ! \Gamma(\nu+n+1)}\left(\frac{z}{2}\right)^{2 n+\nu}, \quad z \in \mathbb{C} .
$$

The rising factorial, or say, the Pochhammer symbol, of $\alpha \in \mathbb{C}$ is defined [12, p. 7497] by

$$
(\alpha)_{n}=\prod_{k=0}^{n-1}(\alpha+k)= \begin{cases}\alpha(\alpha+1) \cdots(\alpha+n-1), & n \geq 1 ; \\ 1, & n=0 .\end{cases}
$$

In [27, p. $377,(3.5)]$ and [28, pp. 109-110, Lemma 1], it was obtained that

$$
I_{\mu}(x) I_{\nu}(x)=\frac{1}{\Gamma(\mu+1) \Gamma(\nu+1)} \sum_{n=0}^{\infty} \frac{(\mu+\nu+n+1)_{n}}{n !(\mu+1)_{n}(\nu+1)_{n}}\left(\frac{x}{2}\right)^{2 n+\mu+\nu} .
$$

2020 Mathematics Subject Classification. Primary 41A58; Secondary 11B73, 11B83, 26A24, 33B10.

Key words and phrases. Maclaurin's series expansion; real power; inverse cosine function; inverse hyperbolic cosine function; inverse sine function; inverse hyperbolic sine function; Stirling number of the first kind; Bell polynomial of the second kind; Faà di Bruno formula; central binomial coefficient; combinatorial identity; infinite series representation; Pi.

This paper was typeset using $\mathcal{A} \mathcal{M} \mathcal{S}-\mathrm{LAT}_{\mathrm{E}} \mathrm{X}$. 
In [4, p. 310], there exists the power series expansion

$$
\left[I_{\nu}(z)\right]^{2}=\sum_{k=0}^{\infty} \frac{1}{[\Gamma(\nu+k+1)]^{2}}\left(\begin{array}{c}
2 k+2 \nu \\
k
\end{array}\right)\left(\frac{z}{2}\right)^{2 k+2 \nu} .
$$

More generally, the series expansions of the functions $\left[I_{\nu}(z)\right]^{r}$ for $\nu \in \mathbb{C} \backslash\{-1,-2, \ldots\}$ and $r, z \in \mathbb{C}$ have been surveyed and investigated in $3,4,13,14,16$. One of the reasons why ones investigated the series expansions of the functions $\left[I_{\nu}(z)\right]^{r}$ is that the products of the (modified) Bessel functions of the first kind appear occasionally in problems of statistical mechanics and plasma physics [2, 17, 18.

In the articles [5, 6, 11, 12, 20, 24, 25], Maclaurin's series expansions of the powers

$$
\begin{aligned}
& \sin ^{m} z, \quad \cos ^{m} z, \quad \tan ^{m} z, \quad \cot ^{m} z, \quad \sec ^{m} z, \quad \csc ^{m} z, \quad(\arctan z)^{m}, \quad(\operatorname{arctanh} z)^{m}, \\
&\left(\frac{\arcsin z}{z}\right)^{m}, \quad \frac{(\arcsin z)^{m}}{\sqrt{1-z^{2}}}, \quad\left(\frac{\operatorname{arcsinh} z}{z}\right)^{m}, \frac{(\operatorname{arcsinh} z)^{m}}{\sqrt{1+z^{2}}}
\end{aligned}
$$

for $m \geq 2$ and their history were reviewed, established, discussed, and applied. Now we recite several nice series expansions as follows.

Theorem 1.1 ([11, Theorem 2.1]). For $m \in \mathbb{N}$ and $|x|<1$, the function $\left(\frac{\arcsin x}{x}\right)^{m}$, whose value at $x=0$ is defined to be 1 , has the nice Maclaurin series expansion

$$
\left(\frac{\arcsin x}{x}\right)^{m}=1+\sum_{k=1}^{\infty} \frac{(-1)^{k}}{\left(\begin{array}{c}
m+2 k \\
m
\end{array}\right)}\left[\sum_{\ell=0}^{2 k}\left(\begin{array}{c}
m+\ell-1 \\
m-1
\end{array}\right) s(m+2 k-1, m+\ell-1)\left(\frac{m+2 k-2}{2}\right)^{\ell}\right] \frac{(2 x)^{2 k}}{(2 k) !}
$$

where $s(n, k)$ denotes the first kind Stirling numbers which can be analytically computed by

$$
|s(n+1, m+1)|=n ! \sum_{\ell_{1}=m}^{n} \frac{1}{\ell_{1}} \sum_{\ell_{2}=m-1}^{\ell_{1}-1} \frac{1}{\ell_{2}} \cdots \sum_{\ell_{m-1}=2}^{\ell_{m-2}-1} \frac{1}{\ell_{m-1}} \sum_{\ell_{m}=1}^{\ell_{m-1}-1} \frac{1}{\ell_{m}}, \quad n \geq m \geq 1 .
$$

Theorem 1.2 ([11, Theorem 5.1]). For $m \in \mathbb{N}$ and $|x|<\infty$, the function $\left(\frac{\operatorname{arcsinh} x}{x}\right)^{m}$, whose value at $x=0$ is defined to be 1 , has the nice Maclaurin series expansion

$$
\left(\frac{\operatorname{arcsinh} x}{x}\right)^{m}=1+\sum_{k=1}^{\infty} \frac{1}{\left(\begin{array}{c}
m+2 k \\
m
\end{array}\right)}\left[\sum_{\ell=0}^{2 k}\left(\begin{array}{c}
m+\ell-1 \\
m-1
\end{array}\right) s(m+2 k-1, m+\ell-1)\left(\frac{m+2 k-2}{2}\right)^{\ell}\right] \frac{(2 x)^{2 k}}{(2 k) !}
$$

where $s(n, k)$ stands for the first kind Stirling numbers generated by 1.5$)$. where $s(n, m)$ denotes the Stirling numbers of the first kind which can be analytically generated by

$$
\frac{[\ln (1+x)]^{m}}{m !}=\sum_{n=m}^{\infty} s(n, m) \frac{x^{n}}{n !}, \quad|x|<1 .
$$

In 11, 12, the series expansion (1.2) was applied to

(1) derive closed-form formulas for special values of the Bell polynomials of the second kind, these closed-form formulas were asked in [19] when studying Grothendieck's inequality and completely correlation preserving functions;

(2) establish series representations of the generalized logsine function, these series representations were considered in $[9,15]$.

The formula 1.3 is a reformulation of [21, Corollary 2.3] and the generating function 1.5 can be found in [26, p. 20, (1.30)].

The series expansions 1.2 and 1.4 in Theorems 1.1 and 1.2 were also recovered in [23, Section 3].

Theorem 1.3 (23, Theorem 4.1]). For $|x|<1$, we have

$$
\frac{(\arccos x)^{2}}{2 !}=\sum_{m=0}^{\infty} \frac{m !}{(2 m+1) ! !} \frac{(1-x)^{m+1}}{m+1}
$$

and

$$
\frac{(\operatorname{arccosh} x)^{2}}{2 !}=-\sum_{m=0}^{\infty} \frac{m !}{(2 m+1) ! !} \frac{(1-x)^{m+1}}{m+1} .
$$


For $k \geq 2$ and $|x|<1$, we have

$$
\frac{(\arccos x)^{2 k}}{(2 k) !}=\sum_{m=0}^{\infty}(-1)^{m}\left[\sum_{\ell=0}^{2 m}\left(\begin{array}{c}
\ell+2 k-1 \\
2 k-1
\end{array}\right) s(2 m+2 k-1, \ell+2 k-1)(m+k-1)^{\ell}\right] \frac{[2(1-x)]^{m+k}}{[2(m+k)] !}
$$

and

$$
\frac{(\operatorname{arccosh} x)^{2 k}}{(2 k) !}=\sum_{m=0}^{\infty}(-1)^{m+k}\left[\sum_{\ell=0}^{2 m}\left(\begin{array}{c}
\ell+2 k-1 \\
2 k-1
\end{array}\right) s(2 m+2 k-1, \ell+2 k-1)(m+k-1)^{\ell}\right] \frac{[2(1-x)]^{m+k}}{[2(m+k)] !}
$$

The series expansions (1.6), (1.7), (1.8), and (1.9) in Theorem 1.3 are Taylor's series expansions around the point $x=1$ of even powers of the inverse cosine function $\arccos x$ and the inverse hyperbolic cosine function $\operatorname{arccosh} x$ in terms of the Stirling numbers of the first kind $s(n, m)$.

What are Maclaurin's series expansions around the point $x=0$ for the power functions $(\arccos x)^{\alpha}$ and $(\operatorname{arccosh} x)^{\alpha}$ with $\alpha \in \mathbb{R}$ ? In Section 3 of this paper, we will answer this interesting and significant question. Besides this, we also

(1) establish explicit formulas for special values of the Bell polynomials of the second kind with respect to the specific sequence $1,0,1,0,9,0,225, \ldots$; see Theorem 2.1 below;

(2) apply two different series expansions of the square function $(\arccos x)^{2}$, including Taylor's series expansion 1.6 in Theorem 1.3, to find infinite series representations of $\pi$ and $\pi^{2}$ respectively; see Theorem 5.1 below;

(3) apply two different series expansions of $(\arccos x)^{2}$ to derive two combinatorial identities involving central binomial coefficients, see Theorem 6.1 below.

\section{EXPlicit formulas for SPECIAL VAlues of Bell POLYNOMials of SECOND Kind}

In [7, Definition 11.2] and [8, p. 134, Theorem A], the Bell polynomials of the second kind, denoted by $\mathrm{B}_{n, k}\left(x_{1}, x_{2}, \ldots, x_{n-k+1}\right)$ for $n \geq k \geq 0$, are defined by

$$
\mathrm{B}_{n, k}\left(x_{1}, x_{2}, \ldots, x_{n-k+1}\right)=\sum_{\substack{1 \leq i \leq n-k+1 \\ \ell_{i} \in\{0\} \cup \mathbb{N} \\ \sum_{i=k+1}^{n-k+1} i \ell_{i}=n \\ \sum_{i=1}^{n-k+1} \ell_{i}=k}} \frac{n !}{\prod_{i=1}^{n-k+1} \ell_{i} !} \prod_{i=1}^{n-k+1}\left(\frac{x_{i}}{i !}\right)^{\ell_{i}} .
$$

The Faà di Bruno formula [7, Theorem 11.4] and [8, p. 139, Theorem C] can be described in terms of $\mathrm{B}_{k, \ell}\left(x_{1}, x_{2}, \ldots, x_{k-\ell+1}\right)$ by

$$
\frac{\mathrm{d}^{k}}{\mathrm{~d} x^{k}} f \circ h(x)=\sum_{\ell=1}^{k} f^{(\ell)}(h(x)) \mathrm{B}_{k, \ell}\left(h^{\prime}(x), h^{\prime \prime}(x), \ldots, h^{(k-\ell+1)}(x)\right), \quad k \in \mathbb{N} .
$$

To establish Maclaurin's series expansions around the point $x=0$ for real powers of the inverse cosine function $\arccos x$ and the inverse hyperbolic cosine function $\operatorname{arccosh} x$, we need the following explicit formulas for special values of the Bell polynomials of the second kind with respect to the sequence

$$
1,0,1,0,9,0,225,0, \ldots, 0,[(2 r-3) ! !]^{2}, 0,[(2 r-1) ! !]^{2}, \ldots
$$

for $r \geq 0$.

Theorem 2.1. For $r, k \in \mathbb{N}$, we have

$$
\begin{aligned}
& \mathrm{B}_{2 r+k, k}\left(1,0,1,0,9,0,225,0, \ldots,[(2 r-3) ! !]^{2}, 0,[(2 r-1) ! !]^{2}\right) \\
&=(-1)^{r} 2^{2 r} \sum_{\ell=0}^{2 r}\left(\begin{array}{c}
k+\ell-1 \\
k-1
\end{array}\right) s(k+2 r-1, k+\ell-1)\left(\frac{k+2 r-2}{2}\right)^{\ell}
\end{aligned}
$$

and

$$
\mathrm{B}_{2 r+k-1, k}\left(1,0,1,0,9,0,225,0, \ldots,[(2 r-3) ! !]^{2}, 0\right)=0,
$$

where $(-1) ! !=0 ! !=1$. 
Proof. In [10, p. 60, 1.641], there is the series expansion

$$
\arccos x=\frac{\pi}{2}-\sum_{\ell=0}^{\infty} \frac{(2 \ell-1) ! !}{(2 \ell) ! !} \frac{x^{2 \ell+1}}{2 \ell+1}, \quad|x|<1 .
$$

The series expansion 2.5 means that

$$
\left.(\arccos x)^{(2 \ell)}\right|_{x=0}=0 \quad \text { and }\left.\quad(\arccos x)^{(2 \ell-1)}\right|_{x=0}=-[(2 \ell-3) ! !]^{2}
$$

for $\ell \in \mathbb{N}$.

At the end of [8, p. 133], there is the formula

$$
\frac{1}{k !}\left(\sum_{m=1}^{\infty} x_{m} \frac{t^{m}}{m !}\right)^{k}=\sum_{n=k}^{\infty} \mathrm{B}_{n, k}\left(x_{1}, x_{2}, \ldots, x_{n-k+1}\right) \frac{t^{n}}{n !}
$$

for $k \geq 0$. Making use of the formula 2.7 yields

$$
\mathrm{B}_{n+k, k}\left(x_{1}, x_{2}, \ldots, x_{n+1}\right)=\left(\begin{array}{c}
n+k \\
k
\end{array}\right) \lim _{t \rightarrow 0} \frac{\mathrm{d}^{n}}{\mathrm{~d} t^{n}}\left[\sum_{m=0}^{\infty} \frac{x_{m+1}}{(m+1) !} t^{m}\right]^{k}, \quad n \geq k \geq 0 .
$$

Taking $x_{m}=\left.(\arccos x)^{(m)}\right|_{x=0}$ for $m \geq 1$ in 2.8), employing the values in 2.6), and utilizing the series expansion 1.2 in Theorem 1.1 give

$$
\begin{aligned}
& \mathrm{B}_{n+k, k}\left(\left.(\arccos x)^{\prime}\right|_{x=0},\left.(\arccos x)^{\prime \prime}\right|_{x=0}, \ldots,\left.(\arccos x)^{(n+1)}\right|_{x=0}\right) \\
= & \mathrm{B}_{n+k, k}\left(-1,0,-1,0,-9,0,-225, \ldots,-\frac{1-(-1)^{n+1}}{2}[(n-1) ! !]^{2}\right) \\
= & \left(\begin{array}{c}
n+k \\
k
\end{array}\right) \lim _{t \rightarrow 0} \frac{\mathrm{d}^{n}}{\mathrm{~d} t^{n}}\left[\sum_{m=0}^{\infty} \frac{\left.(\arccos x)^{(m+1)}\right|_{x=0}}{(m+1) !} t^{m}\right]^{k} \\
= & (-1)^{k}\left(\begin{array}{c}
n+k \\
k
\end{array}\right) \lim _{t \rightarrow 0} \frac{\mathrm{d}^{n}}{\mathrm{~d} t^{n}}\left[\frac{1}{t} \sum_{m=1}^{\infty} \frac{\left.(\arcsin x)^{(m)}\right|_{x=0}}{m !} t^{m}\right]^{k} \\
= & (-1)^{k}\left(\begin{array}{c}
n+k \\
k
\end{array}\right) \lim _{t \rightarrow 0} \frac{\mathrm{d}^{n}}{\mathrm{~d} t^{n}}\left(\frac{\arcsin t}{t}\right)^{k} \\
= & (-1)^{k}\left(\begin{array}{c}
n+k \\
k
\end{array}\right) \lim _{t \rightarrow 0} \frac{\mathrm{d}^{n}}{\mathrm{~d} t^{n}} \sum_{q=1}^{\infty} \frac{(-1)^{q}}{\left(\begin{array}{c}
k+2 q \\
k
\end{array}\right)}\left[\sum_{\ell=0}^{2 q}\left(\begin{array}{c}
k+\ell-1 \\
k-1
\end{array}\right) s(k+2 q-1, k+\ell-1)\left(\frac{k+2 q-2}{2}\right)^{\ell}\right] \frac{(2 t)^{2 q}}{(2 q) !} \\
= & (-1)^{k}\left(\begin{array}{c}
n+k \\
k
\end{array}\right) \lim _{t \rightarrow 0} \sum_{q=1}^{\infty} \frac{(-4)^{q}}{\left(\begin{array}{c}
k+2 q \\
k
\end{array}\right)}\left[\sum_{\ell=0}^{2 q}\left(\begin{array}{c}
k+\ell-1 \\
k-1
\end{array}\right) s(k+2 q-1, k+\ell-1)\left(\frac{k+2 q-2}{2}\right)^{\ell}\right]\langle 2 q\rangle_{n} \frac{t^{2 q-n}}{(2 q) !} \\
= & \left\{\begin{array}{c}
(-1)^{k+r} 2^{2 r} \sum_{\ell=0}^{2 r}\left(\begin{array}{c}
k+\ell-1 \\
k-1
\end{array}\right) s(k+2 r-1, k+\ell-1)\left(\frac{k+2 r-2}{2}\right)^{\ell}, \quad n=2 r \\
0,
\end{array} \quad \begin{array}{c}
n=2 r-1 \\
{ }_{\ell}(k+2 r
\end{array}\right.
\end{aligned}
$$

for $r \in \mathbb{N}$, where the falling factorial $\langle z\rangle_{k}$ of $z \in \mathbb{C}$ is defined by

$$
\langle z\rangle_{k}=\prod_{\ell=0}^{k-1}(z-\ell)= \begin{cases}z(z-1) \cdots(z-k+1), & k \geq 1 ; \\ 1, & k=0 .\end{cases}
$$

Further employing the identity

$$
\mathrm{B}_{n, k}\left(a b x_{1}, a b^{2} x_{2}, \ldots, a b^{n-k+1} x_{n-k+1}\right)=a^{k} b^{n} \mathrm{~B}_{n, k}\left(x_{1}, x_{2}, \ldots, x_{n-k+1}\right)
$$

for $n \geq k \geq 0$ and $a, b \in \mathbb{C}$ in [7, p. 412] and [8, p. 135] and simplifying result in the formulas 2.3. and (2.4). The proof of Theorem 2.1 is complete. 


\section{MACLAURIN'S SERIES EXPANSIONS FOR REAL POWERS OF INVERSE COSINE FUNCTION}

In his section, by means of the Faà di Bruno formula (2.1), with the help of explicit formulas (2.3) and (2.4) in Theorem 2.1, and by virtue of two combinatorial identities in [23, Lemmas 2.1 and 2.2], we establish Maclaurin's series expansions at the point $x=0$ for $(\arccos x)^{\alpha}$ and $(\operatorname{arccosh} x)^{\alpha}$ with $\alpha \in \mathbb{R}$.

Theorem 3.1. For $\alpha \in \mathbb{R}$ and $|x|<1$, we have

$$
\begin{aligned}
& \left(\frac{2 \arccos x}{\pi}\right)^{\alpha}=1+\frac{\langle\alpha\rangle_{2}}{\pi^{2}} \frac{(2 x)^{2}}{2 !} \\
& +\sum_{r=2}^{\infty}(-1)^{r}\left[\sum_{\ell=1}^{r}(-1)^{\ell} \frac{\langle\alpha\rangle_{2 \ell}}{\pi^{2 \ell}} \sum_{q=0}^{2 r-2 \ell}\left(\begin{array}{c}
2 \ell+q-1 \\
2 \ell-1
\end{array}\right) s(2 r-1,2 \ell+q-1)(r-1)^{q}\right] \frac{(2 x)^{2 r}}{(2 r) !} \\
& +\sum_{r=1}^{\infty}(-1)^{r}\left[\sum_{\ell=1}^{r}(-1)^{\ell-1} \frac{\langle\alpha\rangle_{2 \ell-1}}{\pi^{2 \ell-1}} \sum_{q=0}^{2 r-2 \ell}\left(\begin{array}{c}
2 \ell+q-2 \\
2 \ell-2
\end{array}\right) s(2 r-2,2 \ell+q-2)\left(\frac{2 r-3}{2}\right)^{q}\right] \frac{(2 x)^{2 r-1}}{(2 r-1) !},
\end{aligned}
$$

where $\langle\alpha\rangle_{r}$ for $\alpha \in \mathbb{R}$ and $r \in \mathbb{N}$ stands for the falling factorials defined by (2.9) and $s(n, m)$ for $n \geq m \geq 0$ denotes the Stirling numbers of the first kind generated in (1.5).

Proof. Let $u=u(x)=\arccos x$. It is clear that $u=u(x)=\arccos x \rightarrow \frac{\pi}{2}$ as $x \rightarrow 0$. By means of the Faà di Bruno formula 2.1 and the values in 2.6), we obtain

$$
\begin{aligned}
\frac{\mathrm{d}^{k}\left[(\arccos x)^{\alpha}\right]}{\mathrm{d} x^{k}} & =\sum_{\ell=1}^{k} \frac{\mathrm{d}^{\ell}\left(u^{\alpha}\right)}{\mathrm{d} u^{\ell}} \mathrm{B}_{k, \ell}\left((\arccos x)^{\prime},(\arccos x)^{\prime \prime}, \ldots,(\arccos x)^{(k-\ell+1)}\right) \\
& =\sum_{\ell=1}^{k}\langle\alpha\rangle_{\ell} u^{\alpha-\ell} \mathrm{B}_{k, \ell}\left((\arccos x)^{\prime},(\arccos x)^{\prime \prime}, \ldots,(\arccos x)^{(k-\ell+1)}\right) \\
& \rightarrow \sum_{\ell=1}^{k}\langle\alpha\rangle_{\ell}\left(\frac{\pi}{2}\right)^{\alpha-\ell} \mathrm{B}_{k, \ell}\left(-1,0,-1,0,-9, \ldots,-\frac{1-(-1)^{k-\ell+1}}{2}[(k-\ell-1) ! !]^{2}\right)
\end{aligned}
$$

as $x \rightarrow 0$ for $k \in \mathbb{N}$.

When $k=2 r$ and $r \geq 2$, it follows that

$$
\begin{aligned}
\lim _{x \rightarrow 0} \frac{\mathrm{d}^{2 r}\left[(\arccos x)^{\alpha}\right]}{\mathrm{d} x^{2 r}} & =\sum_{\ell=1}^{2 r}\langle\alpha\rangle_{\ell}\left(\frac{\pi}{2}\right)^{\alpha-\ell} \mathrm{B}_{2 r, \ell}\left(-1,0,-1,0,-9, \ldots,-\frac{1-(-1)^{1-\ell}}{2}[(2 r-\ell-1) ! !]^{2}\right) \\
= & \sum_{\ell=1}^{r}\langle\alpha\rangle_{2 \ell}\left(\frac{\pi}{2}\right)^{\alpha-2 \ell} \mathrm{B}_{2 r, 2 \ell}\left(1,0,1,0,9,0,225, \ldots, 0,[(2 r-2 \ell-1) ! !]^{2}\right) \\
= & \sum_{\ell=1}^{r}(-1)^{r-\ell}\langle\alpha\rangle_{2 \ell} \pi^{\alpha-2 \ell} 2^{2 r-\alpha} \sum_{q=0}^{2 r-2 \ell}\left(\begin{array}{c}
2 \ell+q-1 \\
2 \ell-1
\end{array}\right) s(2 r-1,2 \ell+q-1)(r-1)^{q},
\end{aligned}
$$

where we used the identity 2.10 and Theorem 2.1

It is easy to see that

$$
\lim _{x \rightarrow 0} \frac{\mathrm{d}^{2}\left[(\arccos x)^{\alpha}\right]}{\mathrm{d} x^{2}}=(\alpha-1) \alpha\left(\frac{\pi}{2}\right)^{\alpha-2} .
$$

When $k=2 r-1$ for $r \geq 2$, it follows that

$$
\begin{aligned}
\lim _{x \rightarrow 0} & \frac{\mathrm{d}^{2 r-1}\left[(\arccos x)^{\alpha}\right]}{\mathrm{d} x^{2 r-1}}=\sum_{\ell=1}^{2 r-1}(-1)^{\ell}\langle\alpha\rangle_{\ell}\left(\frac{\pi}{2}\right)^{\alpha-\ell} \mathrm{B}_{2 r-1, \ell}\left(1,0,1,0,9, \ldots, \frac{1-(-1)^{\ell}}{2}[(2 r-\ell-2) ! !]^{2}\right) \\
& =-\sum_{\ell=1}^{r}\langle\alpha\rangle_{2 \ell-1}\left(\frac{\pi}{2}\right)^{\alpha-2 \ell+1} \mathrm{~B}_{2 r-1,2 \ell-1}\left(1,0,1,0,9, \ldots,[(2 r-2 \ell-1) ! !]^{2}\right) \\
& =\sum_{\ell=1}^{r}(-1)^{r-\ell-1}\langle\alpha\rangle_{2 \ell-1} \pi^{\alpha-2 \ell+1} 2^{2 r-\alpha-1} \sum_{q=0}^{2 r-2 \ell}\left(\begin{array}{c}
2 \ell+q-2 \\
2 \ell-2
\end{array}\right) s(2 r-2,2 \ell+q-2)\left(\frac{2 r-3}{2}\right)^{q},
\end{aligned}
$$

where we used the identity 2.10 and Theorem 2.1 
It is easy to see that

$$
\lim _{x \rightarrow 0} \frac{\mathrm{d}\left[(\arccos x)^{\alpha}\right]}{\mathrm{d} x}=-\alpha\left(\frac{\pi}{2}\right)^{\alpha-1} .
$$

Combining these four limits 3.2 , 3.3, 3.4, and 3.5) and simplifying yield the series expansion 3.1. The proof of Theorem 3.1 is complete.

Corollary 3.1. For $|x|<1$, we have

$$
\left(\frac{2 \arccos x}{\pi}\right)^{2}=1-\frac{4}{\pi} \sum_{r=1}^{\infty}[(2 r-3) ! !]^{2} \frac{x^{2 r-1}}{(2 r-1) !}+\frac{8}{\pi^{2}} \sum_{r=1}^{\infty}[(2 r-2) ! !]^{2} \frac{x^{2 r}}{(2 r) !} .
$$

Proof. Setting $\alpha=2$ in the series expansion (3.1) in Theorem 3.1 arrives at

$$
\begin{aligned}
\left(\frac{2 \arccos x}{\pi}\right)^{2}= & 1+\frac{2}{\pi^{2}} \frac{(2 x)^{2}}{2 !}+\sum_{r=2}^{\infty}(-1)^{r+1}\left[\frac{2}{\pi^{2}} \sum_{q=0}^{2 r-2}(q+1) s(2 r-1, q+1)(r-1)^{q}\right] \frac{(2 x)^{2 r}}{(2 r) !} \\
& +\sum_{r=1}^{\infty}(-1)^{r}\left[\frac{2}{\pi} \sum_{q=0}^{2 r-2} s(2 r-2, q)\left(\frac{2 r-3}{2}\right)^{q}\right] \frac{(2 x)^{2 r-1}}{(2 r-1) !}
\end{aligned}
$$

Further using the identities

$$
\sum_{\ell=0}^{2 k}(\ell+1) s(2 k+1, \ell+1) k^{\ell}=(-1)^{k}(k !)^{2}, \quad k \in \mathbb{N}
$$

and

$$
\sum_{\ell=0}^{2 k} s(2 k, \ell)\left(k-\frac{1}{2}\right)^{\ell}=(-1)^{k}\left[\frac{(2 k-1) ! !}{2^{k}}\right]^{2}, \quad k \in \mathbb{N}
$$

in 23, Lemmas 2.1 and 2.2], we acquire

$$
\left(\frac{2 \arccos x}{\pi}\right)^{2}=1+\frac{2}{\pi^{2}} \frac{(2 x)^{2}}{2 !}+\frac{2}{\pi^{2}} \sum_{r=2}^{\infty}[(r-1) !]^{2} \frac{(2 x)^{2 r}}{(2 r) !}-\frac{2}{\pi} \sum_{r=1}^{\infty}\left[\frac{(2 r-3) ! !}{2^{r-1}}\right]^{2} \frac{(2 x)^{2 r-1}}{(2 r-1) !} .
$$

The series expansion (3.6) follows. The proof of Corollary 3.1 is complete.

Corollary 3.2. For $k \in \mathbb{N}$ and $|x|<1$, we have

$$
\left(\frac{2 \operatorname{arccosh} x}{\pi}\right)^{2}=-1+\frac{4}{\pi} \sum_{r=1}^{\infty}[(2 r-3) ! !]^{2} \frac{x^{2 r-1}}{(2 r-1) !}-\frac{8}{\pi^{2}} \sum_{r=1}^{\infty}[(2 r-2) ! !]^{2} \frac{x^{2 r}}{(2 r) !}
$$

and

$$
\begin{aligned}
& (-1)^{k}\left(\frac{2 \operatorname{arccosh} x}{\pi}\right)^{2 k}=1+\frac{\langle 2 k\rangle_{2}}{\pi^{2}} \frac{(2 x)^{2}}{2 !} \\
& +\sum_{r=2}^{\infty}(-1)^{r}\left[\sum_{\ell=1}^{r}(-1)^{\ell} \frac{\langle 2 k\rangle_{2 \ell}}{\pi^{2 \ell}} \sum_{q=0}^{2 r-2 \ell}\left(\begin{array}{c}
2 \ell+q-1 \\
2 \ell-1
\end{array}\right) s(2 r-1,2 \ell+q-1)(r-1)^{q}\right] \frac{(2 x)^{2 r}}{(2 r) !} \\
& +\sum_{r=1}^{\infty}(-1)^{r}\left[\sum_{\ell=1}^{r}(-1)^{\ell-1} \frac{\langle 2 k\rangle_{2 \ell-1}}{\pi^{2 \ell-1}} \sum_{q=0}^{2 r-2 \ell}\left(\begin{array}{c}
2 \ell+q-2 \\
2 \ell-2
\end{array}\right) s(2 r-2,2 \ell+q-2)\left(\frac{2 r-3}{2}\right)^{q}\right] \frac{(2 x)^{2 r-1}}{(2 r-1) !}
\end{aligned}
$$

where the falling factorials $\langle 2 k\rangle_{r}$ for $k, r \in \mathbb{N}$ are defined by 2.9 . .

Proof. This follows from substituting the relation $\arccos x=-i \operatorname{arccosh} x$ into the series expansions (3.6) and (3.1) for $\alpha=2 k$. 
4. MACLAURIN'S SERIES EXPANSIONS FOR REAL POWERS OF INVERSE SINE FUNCTION

Theorem 4.1. For $k, n \geq 0$ such that $2 n+1 \geq k \geq 0$, we have

$$
\mathrm{B}_{2 n+1, k}\left(\left.\left(\frac{\arcsin x}{x}\right)^{\prime}\right|_{x=0},\left.\left(\frac{\arcsin x}{x}\right)^{\prime \prime}\right|_{x=0}, \ldots,\left.\left(\frac{\arcsin x}{x}\right)^{(2 n-k+2)}\right|_{x=0}\right)=0 .
$$

For $k, n \in \mathbb{N}$ such that $2 n \geq k \in \mathbb{N}$, we have

$$
\begin{aligned}
& \mathrm{B}_{2 n, k}\left(\left.\left(\frac{\arcsin x}{x}\right)^{\prime}\right|_{x=0},\left.\left(\frac{\arcsin x}{x}\right)^{\prime \prime}\right|_{x=0}, \ldots,\left.\left(\frac{\arcsin x}{x}\right)^{(2 n-k+1)}\right|_{x=0}\right) \\
& =(-1)^{n+k} \frac{(4 n) ! !}{(2 n+k) !} \sum_{q=1}^{k}(-1)^{q}\left(\begin{array}{c}
2 n+k \\
k-q
\end{array}\right) \sum_{\ell=0}^{2 n}\left(\begin{array}{c}
q+\ell-1 \\
q-1
\end{array}\right) s(2 n+q-1, q+\ell-1)\left(\frac{2 n+q-2}{2}\right)^{\ell},
\end{aligned}
$$

where $s(n, k)$ denotes the first kind Stirling numbers generated by 1.5.

Proof. It is well-known that

$$
\arcsin x=\sum_{\ell=0}^{\infty}(2 \ell-1) ! ! \frac{x^{2 \ell+1}}{(2 \ell+1) !}, \quad|x|<1 .
$$

Then

$$
\frac{\arcsin x}{x}=\sum_{\ell=0}^{\infty} \frac{(2 \ell-1) ! !}{2 \ell+1} \frac{x^{2 \ell}}{(2 \ell) !}, \quad|x|<1
$$

Therefore, for $\ell \in \mathbb{N}$, we have

$$
\left.\left(\frac{\arcsin x}{x}\right)^{(\ell)}\right|_{x=0}= \begin{cases}0, & \ell=2 m+1 \\ \frac{(2 m-1) ! !}{2 m+1}, & \ell=2 m\end{cases}
$$

for $m \geq 0$. Then it is easy to see that

$$
\begin{aligned}
& \mathrm{B}_{n, k}\left(\left.\left(\frac{\arcsin x}{x}\right)^{\prime}\right|_{x=0},\left.\left(\frac{\arcsin x}{x}\right)^{\prime \prime}\right|_{x=0}, \ldots,\left.\left(\frac{\arcsin x}{x}\right)^{(n-k+1)}\right|_{x=0}\right) \\
& =\mathrm{B}_{n, k}\left(0, \frac{1}{3}, 0, \frac{9}{5}, 0, \frac{225}{7}, 0,1225,0, \ldots, \frac{1+(-1)^{n-k+1}}{2} \frac{[(n-k) ! !]^{2}}{n-k+2}\right) .
\end{aligned}
$$

Consequently, from [11, Theorem 3.1] and [12, Theorem 1.1], we can turn out the formulas (4.1) and (4.2). The proof of Theorem 4.1 is complete.

Theorem 4.2. For $\alpha \in \mathbb{R}$ and $|x|<1$, we have

$$
\begin{aligned}
\left(\frac{\arcsin x}{x}\right)^{\alpha}=1+\sum_{n=1}^{\infty}(-1)^{n}(4 n) ! ! & {\left[\sum_{k=1}^{2 n}(-1)^{k} \frac{\langle\alpha\rangle_{k}}{(2 n+k) !} \sum_{q=1}^{k}(-1)^{q}\left(\begin{array}{c}
2 n+k \\
k-q
\end{array}\right)\right.} \\
& \left.\times \sum_{\ell=0}^{2 n}\left(\begin{array}{c}
q+\ell-1 \\
q-1
\end{array}\right) s(2 n+q-1, q+\ell-1)\left(\frac{2 n+q-2}{2}\right)^{\ell}\right] \frac{x^{2 n}}{(2 n) !} .
\end{aligned}
$$

Proof. By the Faà di Bruno formula (2.1), it follows that

$$
\begin{gathered}
\frac{\mathrm{d}^{n}}{\mathrm{~d} x^{n}}\left[\left(\frac{\arcsin x}{x}\right)^{\alpha}\right]=\sum_{k=0}^{n}\langle\alpha\rangle_{k}\left(\frac{\arcsin x}{x}\right)^{\alpha-k} \\
\times \mathrm{B}_{n, k}\left(\left(\frac{\arcsin x}{x}\right)^{\prime},\left(\frac{\arcsin x}{x}\right)^{\prime \prime}, \ldots,\left(\frac{\arcsin x}{x}\right)^{(n-k+1)}\right) \\
\rightarrow \sum_{k=0}^{n}\langle\alpha\rangle_{k} \mathrm{~B}_{n, k}\left(\left.\left(\frac{\arcsin x}{x}\right)^{\prime}\right|_{x=0},\left.\left(\frac{\arcsin x}{x}\right)^{\prime \prime}\right|_{x=0}, \ldots,\left.\left(\frac{\arcsin x}{x}\right)^{(n-k+1)}\right|_{x=0}\right)
\end{gathered}
$$


as $x \rightarrow 0$. Consequently, we figure out that

$$
\begin{gathered}
\left(\frac{\arcsin x}{x}\right)^{\alpha}=\sum_{n=0}^{\infty} \lim _{x \rightarrow 0} \frac{\mathrm{d}^{2 n}}{\mathrm{~d} x^{2 n}}\left[\left(\frac{\arcsin x}{x}\right)^{\alpha}\right] \frac{x^{2 n}}{(2 n) !} \\
=1+\sum_{n=1}^{\infty}\left[\sum_{k=1}^{2 n}\langle\alpha\rangle_{k} \mathrm{~B}_{2 n, k}\left(\left.\left(\frac{\arcsin x}{x}\right)^{\prime}\right|_{x=0},\left.\left(\frac{\arcsin x}{x}\right)^{\prime \prime}\right|_{x=0}, \ldots,\left.\left(\frac{\arcsin x}{x}\right)^{(2 n-k+1)}\right|_{x=0}\right)\right] \frac{x^{2 n}}{(2 n) !} \\
=1+\sum_{n=1}^{\infty}(-1)^{n}(4 n) ! !\left[\sum_{k=1}^{2 n}(-1)^{k} \frac{\langle\alpha\rangle_{k}}{(2 n+k) !} \sum_{q=1}^{k}(-1)^{q}\left(\begin{array}{c}
2 n+k \\
k-q
\end{array}\right) \sum_{\ell=0}^{2 n}\left(\begin{array}{c}
q+\ell-1 \\
q-1
\end{array}\right)\right. \\
\left.\times s(2 n+q-1, q+\ell-1)\left(\frac{2 n+q-2}{2}\right)^{\ell}\right] \frac{x^{2 n}}{(2 n) !} .
\end{gathered}
$$

The proof of Theorem 4.2 is complete.

\section{Infinite SERIES REPRESEntations RELATED to Pi AND its SQUARE}

By means of comparing the series expansion (1.6) in Theorem 1.3 with the series expansion (3.6) in Corollary 3.1, we can find the following infinite series representations of $\pi$ and $\pi^{2}$.

Theorem 5.1. For $r \in \mathbb{N}$, the constants $\pi$ and $\pi^{2}$ can be represented by

$$
\pi=\frac{2^{2 r-1}}{\left(\begin{array}{c}
2 r-2 \\
r-1
\end{array}\right)} \sum_{m=2 r-1}^{\infty} \frac{2^{m}}{m} \frac{\left(\begin{array}{c}
m-1 \\
2 r-2
\end{array}\right)}{\left(\begin{array}{c}
2 m \\
m
\end{array}\right)}
$$

and

$$
\pi^{2}=8 \sum_{m=1}^{\infty} \frac{2^{m}}{m^{2}} \frac{1}{\left(\begin{array}{c}
2 m \\
m
\end{array}\right)} .
$$

Proof. Maclaurin's series expansion (3.6) can be reformulated as

$$
(\arccos x)^{2}=\frac{\pi^{2}}{4}-\pi \sum_{r=1}^{\infty}[(2 r-3) ! !]^{2} \frac{x^{2 r-1}}{(2 r-1) !}+2 \sum_{r=1}^{\infty}[(2 r-2) ! !]^{2} \frac{x^{2 r}}{(2 r) !}, \quad|x|<1 .
$$

The series expansion 1.6 in Theorem 1.3 can be rearranged as

$$
\begin{aligned}
& \frac{(\arccos x)^{2}}{2 !}=1-x+\sum_{m=1}^{\infty} \frac{m !}{(2 m+1) ! !(m+1)} \sum_{\ell=0}^{m+1}(-1)^{\ell}\left(\begin{array}{c}
m+1 \\
\ell
\end{array}\right) x^{\ell} \\
& =\sum_{m=0}^{\infty} \frac{m !}{(2 m+1) ! !(m+1)}-\left[\sum_{m=0}^{\infty} \frac{m !}{(2 m+1) ! !}\right] x+\sum_{m=1}^{\infty} \frac{m !}{(2 m+1) ! !(m+1)} \sum_{\ell=1}^{m}(-1)^{\ell+1}\left(\begin{array}{c}
m+1 \\
\ell+1
\end{array}\right) x^{\ell+1} \\
& =\sum_{m=0}^{\infty} \frac{m !}{(2 m+1) ! !(m+1)}-\left[\sum_{m=0}^{\infty} \frac{m !}{(2 m+1) ! !}\right] x+\sum_{\ell=2}^{\infty}(-1)^{\ell}\left[\sum_{m=\ell}^{\infty} \frac{(m-1) !}{(2 m-1) ! ! m}\left(\begin{array}{c}
m \\
\ell
\end{array}\right)\right] x^{\ell} \\
& =\sum_{m=0}^{\infty} \frac{2^{m+1}}{(m+1)^{2}} \frac{1}{\left(\begin{array}{c}
2 m+2 \\
m+1
\end{array}\right)}+\sum_{\ell=1}^{\infty}(-1)^{\ell}\left[\sum_{m=\ell}^{\infty} \frac{2^{m}}{m} \frac{\left(\begin{array}{c}
m-1 \\
\ell-1
\end{array}\right)}{\left(\begin{array}{c}
2 m \\
m
\end{array}\right)}\right] \frac{x^{\ell}}{\ell} .
\end{aligned}
$$

Accordingly, we obtain

$$
(\arccos x)^{2}=2 \sum_{m=0}^{\infty} \frac{2^{m+1}}{(m+1)^{2}} \frac{1}{\left(\begin{array}{c}
2 m+2 \\
m+1
\end{array}\right)}+2 \sum_{\ell=1}^{\infty}(-1)^{\ell}\left[\sum_{m=\ell}^{\infty} \frac{2^{m}}{m} \frac{\left(\begin{array}{c}
m-1 \\
\ell-1
\end{array}\right)}{\left(\begin{array}{c}
2 m \\
m
\end{array}\right)}\right] \frac{x^{\ell}}{\ell} .
$$

Comparing the series expansion 5.4 with the series expansion 5.3 produces

$$
\begin{aligned}
& 2 \sum_{m=0}^{\infty} \frac{2^{m+1}}{(m+1)^{2}} \frac{1}{\left(\begin{array}{c}
2 m+2 \\
m+1
\end{array}\right)}=\frac{\pi^{2}}{4}, \\
& 2(-1)^{2 r-1}\left[\sum_{m=2 r-1}^{\infty} \frac{2^{m}}{m} \frac{\left(\begin{array}{c}
m-1 \\
2 r-2
\end{array}\right)}{\left(\begin{array}{c}
2 m \\
m
\end{array}\right)}\right] \frac{x^{2 r-1}}{2 r-1}=-\pi[(2 r-3) ! !]^{2} \frac{x^{2 r-1}}{(2 r-1) !},
\end{aligned}
$$


and

$$
2(-1)^{2 r}\left[\sum_{m=2 r}^{\infty} \frac{2^{m}}{m} \frac{\left(\begin{array}{c}
m-1 \\
2 r-1
\end{array}\right)}{\left(\begin{array}{c}
2 m \\
m
\end{array}\right)}\right] \frac{x^{2 r}}{2 r}=2[(2 r-2) ! !]^{2} \frac{x^{2 r}}{(2 r) !}
$$

for $r \in \mathbb{N}$. The second equation can be rewritten as

$$
\sum_{m=2 r-1}^{\infty} \frac{2^{m}}{m} \frac{\left(\begin{array}{c}
m-1 \\
2 r-2
\end{array}\right)}{\left(\begin{array}{c}
2 m \\
m
\end{array}\right)}=\frac{\pi}{2} \frac{[(2 r-3) ! !]^{2}}{(2 r-2) !}=\pi \frac{\left(\begin{array}{c}
2 r-2 \\
r-1
\end{array}\right)}{2^{2 r-1}}
$$

for $r \in \mathbb{N}$. The series representations (5.1) and 5.2 follows. The proof of Theorem 5.1 is complete.

By taking the special value $x=\frac{1}{2}$ on both sides of 3.1] in Theorem 3.1. we can obtain the following interesting series representation.

Theorem 5.2. For $\alpha \in \mathbb{R}$, we have

$$
\begin{aligned}
\left(\frac{2}{3}\right)^{\alpha} & =1+\sum_{\ell=1}^{\infty}(-1)^{\ell}\left\{\sum_{r=\ell}^{\infty} \frac{(-1)^{r}}{(2 r) !}\left[s(2 r-1,2 \ell-1)+\sum_{q=1}^{2 r-2 \ell}\left(\begin{array}{c}
2 \ell+q-1 \\
2 \ell-1
\end{array}\right) s(2 r-1,2 \ell+q-1)(r-1)^{q}\right]\right\} \frac{\langle\alpha\rangle_{2 \ell}}{\pi^{2 \ell}} \\
& +\sum_{\ell=1}^{\infty}(-1)^{\ell-1}\left[\sum_{r=\ell}^{\infty} \frac{(-1)^{r}}{(2 r-1) !} \sum_{q=0}^{2 r-2 \ell}\left(\begin{array}{c}
2 \ell+q-2 \\
2 \ell-2
\end{array}\right) s(2 r-2,2 \ell+q-2)\left(\frac{2 r-3}{2}\right)^{q}\right] \frac{\langle\alpha\rangle_{2 \ell-1}}{\pi^{2 \ell-1}} .
\end{aligned}
$$

Proof. This follows from taking the special value $x=\frac{1}{2}$ on both sides of 3.1 in Theorem 3.1 and interchanging the orders of sums. The proof of Theorem 5.2 is complete.

Corollary 5.1. The circular constant $\pi$ can be represented as

$$
\frac{\pi}{6}=\sum_{r=1}^{\infty} \frac{[(2 r-3) ! !]^{2}}{(4 r-2) ! !} .
$$

Proof. From taking $\alpha=1$ on both sides of 5.6 in Theorem 5.2 and simplifying, we doscover

$$
\frac{\pi}{3}=\sum_{r=1}^{\infty} \frac{(-1)^{r-1}}{(2 r-1) !} \sum_{q=0}^{2 r-2} s(2 r-2, q)\left(\frac{2 r-3}{2}\right)^{q} .
$$

Further making use of the identity 3.8 and simplifying, we conclude the series representation (5.7). The proof of Corollary 5.1 is complete.

Corollary 5.2 ([23, Theorem 5.1]). The constant $\pi^{2}$ satisfies

$$
\frac{\pi^{2}}{18}=\sum_{r=1}^{\infty} \frac{[(r-1) !]^{2}}{(2 r) !}
$$

Proof. The series representation (5.9) comes from taking $\alpha=2$ on both sides of 5.6 in Theorem 5.2 and simplifying give

$$
\begin{aligned}
\frac{4}{9}= & -\frac{2}{\pi^{2}} \sum_{r=1}^{\infty} \frac{(-1)^{r}}{(2 r) !}\left[s(2 r-1,1)+\sum_{q=1}^{2 r-2}(q+1) s(2 r-1, q+1)(r-1)^{q}\right] \\
& +\frac{2}{\pi} \sum_{r=1}^{\infty} \frac{(-1)^{r}}{(2 r-1) !} \sum_{q=0}^{2 r-2} s(2 r-2, q)\left(\frac{2 r-3}{2}\right)^{q} .
\end{aligned}
$$

Further using the formula $s(r, 1)=(-1)^{r-1}(r-1)$ ! for $r \in \mathbb{N}$, employing the identities (3.7) and 3.8), and simplifying, we acquire

$$
\frac{4}{9}=1-\frac{2}{\pi^{2}} \sum_{r=1}^{\infty} \frac{(-1)^{r}}{(2 r) !}(-1)^{r-1}[(r-1) !]^{2}+\frac{2}{\pi} \sum_{r=1}^{\infty} \frac{(-1)^{r}}{(2 r-1) !}(-1)^{r-1}\left[\frac{(2 r-3) ! !}{2^{r-1}}\right]^{2}
$$

that is,

$$
\frac{4}{9}=1-\frac{4}{\pi} \sum_{r=1}^{\infty} \frac{[(2 r-3) ! !]^{2}}{(4 r-2) ! !}+\frac{2}{\pi^{2}} \sum_{r=1}^{\infty} \frac{[(r-1) !]^{2}}{(2 r) !}
$$


Substituting (5.7) in Corollary 5.1 into (5.9) reveals

$$
\frac{4}{9}=1-\frac{4}{\pi} \frac{\pi}{6}+\frac{2}{\pi^{2}} \sum_{r=1}^{\infty} \frac{[(r-1) !]^{2}}{(2 r) !} .
$$

The series representation 5.8 is thus obtained. The proof of Corollary 5.2 is complete.

\section{Two COMBINATORIAL IDENTITIES}

Squaring on both sides of the equation (2.5) and comparing with Maclaurin's series expansion (3.6) in Corollary 3.1. as well as rewriting the equation 5.5), we can derive the following two combinatorial identities.

Theorem 6.1. For $r \in \mathbb{N}$, we have

$$
\sum_{q=0}^{r-1} \frac{\left(\begin{array}{c}
2 q \\
q
\end{array}\right)}{2 q+1} \frac{\left(\begin{array}{c}
2 r-2 q-2 \\
r-q-1
\end{array}\right)}{2 r-2 q-1}=\frac{2^{4 r-3}}{r^{2}\left(\begin{array}{c}
2 r \\
r
\end{array}\right)}
$$

and

$$
\sum_{m=2 r}^{\infty} \frac{2^{m}}{m} \frac{\left(\begin{array}{c}
m-1 \\
2 r-1
\end{array}\right)}{\left(\begin{array}{c}
2 m \\
m
\end{array}\right)}=\frac{2^{2 r-2}}{2 r-1} \frac{1}{\left(\begin{array}{c}
2 r-2 \\
r-1
\end{array}\right)} .
$$

Proof. From the series expansion 2.5 and Cauchy's product, we conclude that

$$
\begin{gathered}
(\arccos x)^{2}=\left[\frac{\pi}{2}-\sum_{r=0}^{\infty} \frac{(2 r-1) ! !}{(2 r) ! !} \frac{x^{2 r+1}}{2 r+1}\right]^{2} \\
=\left(\frac{\pi}{2}\right)^{2}-\pi \sum_{r=0}^{\infty} \frac{(2 r-1) ! !}{(2 r) ! !} \frac{x^{2 r+1}}{2 r+1}+x^{2}\left[\sum_{r=0}^{\infty} \frac{(2 r-1) ! !}{(2 r) ! !} \frac{x^{2 r}}{2 r+1}\right]^{2} \\
=\left(\frac{\pi}{2}\right)^{2}-\pi \sum_{r=0}^{\infty} \frac{(2 r-1) ! !}{(2 r) ! !} \frac{x^{2 r+1}}{2 r+1}+\sum_{r=0}^{\infty}\left[\sum_{q=0}^{r} \frac{(2 q-1) ! !}{(2 q) ! !(2 q+1)} \frac{(2 r-2 q-1) ! !}{(2 r-2 q) ! !(2 r-2 q+1)}\right] x^{2 r+2} \\
=\left(\frac{\pi}{2}\right)^{2}-\pi \sum_{r=1}^{\infty} \frac{(2 r-3) ! !}{(2 r-2) ! !} \frac{x^{2 r-1}}{2 r-1}+\sum_{r=1}^{\infty}\left[\sum_{q=0}^{r-1} \frac{(2 q-1) ! !}{(2 q) ! !(2 q+1)} \frac{(2 r-2 q-3) ! !}{(2 r-2 q-2) ! !(2 r-2 q-1)}\right] x^{2 r} .
\end{gathered}
$$

Comparing this with (5.3), equating coefficients of the factors $x^{2 r}$, and simplifying reveal the combinatorial identity (6.1).

The equation (5.5) can be simplified as

$$
\sum_{m=2 r}^{\infty} \frac{2^{m}}{m} \frac{\left(\begin{array}{c}
m-1 \\
2 r-1
\end{array}\right)}{\left(\begin{array}{c}
2 m \\
m
\end{array}\right)}=\frac{[(2 r-2) ! !]^{2}}{(2 r-1) !}=\frac{2^{2 r-2}}{2 r-1} \frac{1}{\left(\begin{array}{c}
2 r-2 \\
r-1
\end{array}\right)}, \quad r \in \mathbb{N} .
$$

The combinatorial identity 6.2 is thus proved. The proof of Theorem 6.1 is complete.

\section{REMARKS}

Finally, we give several remarks related to our main results.

Remark 7.1. It is trivial that $\mathrm{B}_{k, k}(x)=x^{k}$ and $\mathrm{B}_{k+1,0}\left(x_{1}, x_{2}, \ldots, x_{k+2}\right)=0$ for $k \geq 0$. Consequently, we considered in Theorem 2.1 all nontrivial cases of $\mathrm{B}_{n, k}\left(x_{1}, x_{2}, \ldots, x_{n-k+1}\right)$ for $n \geq k \geq 0$ with respect to the sequence listed in (2.2), which is equivalent to the sequence

$$
\left.(\arcsin x)^{(k)}\right|_{x=0}=-\left.(\arccos x)^{(k)}\right|_{x=0}, \quad k \in \mathbb{N} .
$$

Using the formulas (2.3) and (2.4) in Theorem 2.1, we can compute Maclaurin's series expansions at $x=0$ for the kind of composite functions $f(\arccos x)$ and $f(\arcsin x)$, such as $(\arcsin x)^{\alpha}$ for $\alpha \in \mathbb{R}$ and $|x|<1$, if the derivatives of $f$ is explicitly computable. 
Remark 7.2. In the series expansion 3.1], if $0^{0}$ is assumed to be 1 , the term $\frac{\langle\alpha\rangle_{2}}{\pi^{2}} \frac{(2 x)^{2}}{2 !}$ can be combined into the first sum in Theorem 3.1. Then the series expansion (3.1) can be reformulated as

$$
\begin{gathered}
\left(\frac{2 \arccos x}{\pi}\right)^{\alpha}=1+\sum_{r=1}^{\infty}(-1)^{r}\left[\sum_{\ell=1}^{r}(-1)^{\ell} \frac{\langle\alpha\rangle_{2 \ell}}{\pi^{2 \ell}} \sum_{q=0}^{2 r-2 \ell}\left(\begin{array}{c}
2 \ell+q-1 \\
2 \ell-1
\end{array}\right) s(2 r-1,2 \ell+q-1)(r-1)^{q}\right] \frac{(2 x)^{2 r}}{(2 r) !} \\
+\sum_{r=1}^{\infty}(-1)^{r}\left[\sum_{\ell=1}^{r}(-1)^{\ell-1} \frac{\langle\alpha\rangle_{2 \ell-1}}{\pi^{2 \ell-1}} \sum_{q=0}^{2 r-2 \ell}\left(\begin{array}{c}
2 \ell+q-2 \\
2 \ell-2
\end{array}\right) s(2 r-2,2 \ell+q-2)\left(\frac{2 r-3}{2}\right)^{q}\right] \frac{(2 x)^{2 r-1}}{(2 r-1) !}
\end{gathered}
$$

for $\alpha \in \mathbb{R}$ and $|x|<1$. Similarly, the series expansion $(3.10)$ in Corollary 3.2 can be reformulated as

$$
\begin{aligned}
&(-1)^{k}\left(\frac{2 \operatorname{arccosh} x}{\pi}\right)^{2 k}= 1+\sum_{r=2}^{\infty}(-1)^{r}\left[\sum_{\ell=1}^{r}(-1)^{\ell} \frac{\langle 2 k\rangle_{2 \ell}}{\pi^{2 \ell}}\right. \\
&\left.\times \sum_{q=0}^{2 r-2 \ell}\left(\begin{array}{c}
\ell \ell+q-1 \\
2 \ell-1
\end{array}\right) s(2 r-1,2 \ell+q-1)(r-1)^{q}\right] \frac{(2 x)^{2 r}}{(2 r) !} \\
&+\sum_{r=1}^{\infty}(-1)^{r}\left[\sum_{\ell=1}^{r}(-1)^{\ell-1} \frac{\langle 2 k\rangle_{2 \ell-1}}{\pi^{2 \ell-1}} \sum_{q=0}^{2 r-2 \ell}\left(\begin{array}{c}
2 \ell+q-2 \\
2 \ell-2
\end{array}\right) s(2 r-2,2 \ell+q-2)\left(\frac{2 r-3}{2}\right)^{q}\right] \frac{(2 x)^{2 r-1}}{(2 r-1) !}
\end{aligned}
$$

for $k \in \mathbb{N}$ and $|x|<1$.

Remark 7.3. Maclaurin's series expansion (3.9) can be reformulated as

$$
(\operatorname{arccosh} x)^{2}=-\frac{\pi^{2}}{4}+\pi \sum_{r=1}^{\infty}[(2 r-3) ! !]^{2} \frac{x^{2 r-1}}{(2 r-1) !}-2 \sum_{r=1}^{\infty}[(2 r-2) ! !]^{2} \frac{x^{2 r}}{(2 r) !}, \quad|x|<1 .
$$

The series expansions (5.3) and (7.1) are more beautiful and concise in form.

Remark 7.4. The relations

and

$$
\arccos x=2 \arctan \sqrt{\frac{1-x}{1+x}}, \quad-1<x \leq 1
$$

$$
\arcsin x=2 \arctan \frac{x}{1+\sqrt{1-x^{2}}}, \quad|x| \leq 1
$$

can be utilized to derive series expansions of powers of the inverse tangent function $\arctan x$ from series expansions (1.6), (1.8), (3.1), (3.6), and (5.3) of powers of the inverse cosine function $\arccos x$.

Remark 7.5. As for the infinite series representations (5.1) and (5.2) in Theorem 5.1 and the series representation (5.7) in Corollary 5.1, we would like to mention the first unsolved problem posed by Herbert Wilf on December 13, 2010 at https://www2.math.upenn.edu/ wilf/website/UnsolvedProblems.pdf, see Figure 1 .

Remark 7.6. This paper is a slightly revised version of the preprint [22].

\section{Declarations}

Acknowledgements: Not applicable.

Availability of data and material: Data sharing is not applicable to this article as no new data were created or analyzed in this study.

Competing interests: The author declares that he has no conflict of competing interests.

Funding: Not applicable.

Authors' contributions: Not applicable.

\section{REFERENCES}

[1] M. Abramowitz and I. A. Stegun (Eds), Handbook of Mathematical Functions with Formulas, Graphs, and Mathematical Tables, National Bureau of Standards, Applied Mathematics Series 55, 10th printing, Washington, 1972.

[2] M. Bakker and N. M. Temme, Sum rule for products of Bessel functions: Comments on a paper by Newberger, J. Math. Phys. 25 (1984), no. 5, 1266-1267; available online at https://doi.org/10.1063/1.526282

[3] Á. Baricz, Powers of modified Bessel functions of the first kind, Appl. Math. Lett. 23 (2010), no. 6, 722-724; available online at https://doi.org/10.1016/j.aml.2010.02.015 


\section{Some unsolved problems}

\section{Herbert Wilf}

December 13, 2010

Here are some mathematical problems that are, as far as I know, unsolved, and which I have encountered in recent work.

\section{Series for $\pi$}

A great many rapidly converging series for $\pi$ are known. Most often they are of the form

$$
\pi=\sum_{n \geq 0} t_{n}
$$

where $t_{n}$ is a hypergeometric term, that is, $t_{n+1} / t_{n}$ is a rational function of $n$. Our question roughly is this - how fast can such a series converge to $\pi$ ?

Of course without further conditions the question is trivial, so we must add that the hypergeometric term $t_{n}$ has rational coefficients. The known series all seem to converge exponentially fast, that is, $C=\lim t_{n}^{1 / n}$ exists and is finite and nonzero. Given any such series it is simple to construct another one in which $C$ is replaced by $C^{2}$, so there exist such representations of $\pi$ in which the constant $C$ is arbitrarily small. But can it be 0 ? That is, can such a series converge superexponentially fast?

We ask the question precisely as follows.

Does there exist an entire function $f(z)=\sum_{n \geq 0} a_{n} z^{n}$ such that the coefficients $a_{n}$ are hypergeometric terms over the rational numbers, and $f(1)=\pi$ ?

It is worth noting that if we replace " $\pi$ " by " $e$ " in the above it becomes quite trivial, since $e^{z}$ is an entire function.

Figure 1. Series for $\pi$

[4] C. M. Bender, D. C. Brody, and B. K. Meister, On powers of Bessel functions, J. Math. Phys. 44 (2003), no. 1, 309-314; available online at https://doi.org/10.1063/1.1526940

[5] J. M. Borwein and M. Chamberland, Integer powers of arcsin, Int. J. Math. Math. Sci. 2007, Art. ID 19381, 10 pages; available online at https://doi.org/10.1155/2007/19381

[6] Yu. A. Brychkov, Power expansions of powers of trigonometric functions and series containing Bernoulli and Euler polynomials, Integral Transforms Spec. Funct. 20 (2009), no. 11-12, 797-804; available online at https://doi.org/10. $1080 / 10652460902867718$

[7] C. A. Charalambides, Enumerative Combinatorics, CRC Press Series on Discrete Mathematics and its Applications. Chapman \& Hall/CRC, Boca Raton, FL, 2002.

[8] L. Comtet, Advanced Combinatorics: The Art of Finite and Infinite Expansions, Revised and Enlarged Edition, D. Reidel Publishing Co., 1974; available online at https://doi.org/10.1007/978-94-010-2196-8

[9] A. I. Davydychev and M. Yu. Kalmykov, New results for the $\varepsilon$-expansion of certain one-, two- and three-loop Feynman diagrams, Nuclear Phys. B 605 (2001), no. 1-3, 266-318; available online at https://doi.org/10.1016/S0550-3213(01) 00095-5

[10] I. S. Gradshteyn and I. M. Ryzhik, Table of Integrals, Series, and Products, Translated from the Russian, Translation edited and with a preface by Daniel Zwillinger and Victor Moll, Eighth edition, Revised from the seventh edition, Elsevier/Academic Press, Amsterdam, 2015; available online at https://doi.org/10.1016/B978-0-12-384933-5.00013-8

[11] B.-N. Guo, D. Lim, and F. Qi, Maclaurin series expansions for powers of inverse (hyperbolic) sine, for powers of inverse (hyperbolic) tangent, and for incomplete gamma functions, with applications, arXiv (2021), available online at https://arxiv.org/abs/2101.10686v6

[12] B.-N. Guo, D. Lim, and F. Qi, Series expansions of powers of arcsine, closed forms for special values of Bell polynomials, and series representations of generalized logsine functions, AIMS Math. 6 (2021), no. 7, 7494-7517; available online at https://doi.org/10.3934/math.2021438

[13] Y. Hong, B.-N. Guo, and F. Qi, Determinantal expressions and recursive relations for the Bessel zeta function and for a sequence originating from a series expansion of the power of modified Bessel function of the first kind, CMES Comput. Model. Eng. Sci. 129 (2021), no. 1, 409-423; available online at https://doi.org/10.32604/cmes.2021.016431

[14] F. T. Howard, Integers related to the Bessel function $J_{1}(z)$, Fibonacci. Quart. 23 (1985), no. 3, 249-257.

[15] M. Yu. Kalmykov and A. Sheplyakov, lsjk-a C++library for arbitrary-precision numeric evaluation of the generalized log-sine functions, Computer Phys. Commun. 172 (2005), no. 1, 45-59; available online at https://doi.org/10.1016/ j.cpc.2005.04.013

[16] V. H. Moll and C. Vignat, On polynomials connected to powers of Bessel functions, Int. J. Number Theory 10 (2014), no. 5, 1245-1257; available online at https://doi.org/10.1142/S1793042114500249 
[17] B. S. Newberger, Erratum: New sum rule for products of Bessel functions with application to plasma physics, J. Math. Phys. 24 (1983), no. 8, 2250-2250; available online at https://doi.org/10.1063/1.525940.

[18] B. S. Newberger, New sum rule for products of Bessel functions with application to plasma physics, J. Math. Phys. 23 (1982), no. 7, 1278-1281; available online at https://doi.org/10.1063/1.525510

[19] F. Oertel, Grothendieck's inequality and completely correlation preserving functions - a summary of recent results and an indication of related research problems, arXiv (2020), available online at https://arxiv.org/abs/2010.00746v2.

[20] F. Qi, Derivatives of tangent function and tangent numbers, Appl. Math. Comput. 268 (2015), 844-858; available online at http://dx.doi.org/10.1016/j.amc.2015.06.123.

[21] F. Qi, Explicit formulas for computing Bernoulli numbers of the second kind and Stirling numbers of the first kind, Filomat 28 (2014), no. 2, 319-327; available online at https://doi.org/10.2298/FIL14023190.

[22] F. Qi, Maclaurin's series expansions of real powers of inverse (hyperbolic) cosine functions and applications, Research Square (2021), available online at https://doi.org/10.21203/rs.3.rs-959177/v1

[23] F. Qi, Taylor's series expansions for even powers of inverse cosine function and series representations for powers of $P i$, arXiv (2021), available online at https://arxiv.org/abs/2110.02749

[24] F. Qi, C.-P. Chen, and D. Lim, Several identities containing central binomial coefficients and derived from series expansions of powers of the arcsine function, Results Nonlinear Anal. 4 (2021), no. 1, 57-64; available online at https://doi.org/10.53006/rna.867047.

[25] F. Qi, D.-W. Niu, D. Lim, and Y.-H. Yao, Special values of the Bell polynomials of the second kind for some sequences and functions, J. Math. Anal. Appl. 491 (2020), no. 2, Article 124382, 31 pages; available online at https://doi.org/ $10.1016 / \mathrm{j} \cdot \mathrm{jmaa} .2020 .124382$

[26] N. M. Temme, Special Functions: An Introduction to Classical Functions of Mathematical Physics, A WileyInterscience Publication, John Wiley \& Sons, Inc., New York, 1996; available online at http://dx.doi.org/10.1002/ 9781118032572

[27] V. R. Thiruvenkatachar and T. S. Nanjundiah, Inequalities concerning Bessel functions and orthogonal polynomials, Proc. Ind. Acad. Sci. Sect. A 33 (1951), 373-384.

[28] Z.-H. Yang and S.-Z. Zheng, Monotonicity and convexity of the ratios of the first kind modified Bessel functions and applications, Math. Inequal. Appl. 21 (2018), no. 1, 107-125; available online at https://doi.org/10.7153/ mia-2018-21-09

Institute of Mathematics, Henan Polytechnic University, Jiaozuo 454010, Henan, China

School of Mathematical Sciences, Tiangong University, Tianjin 300387, China

Email address: qifeng618@gmail.com, qifeng618@hotmail.com, qifeng618@qq.com

$U R L$ : https://qifeng618.wordpress.com, https://orcid.org/0000-0001-6239-2968 